\title{
Avaliação microestrutural de um aço de baixo carbono submetido ao tratamento de boretação pré e pós-soldagem GTAW.
}

\section{Microstructural evaluation of a low carbon steel submitted to boriding treatment pre and post GTAW welding}

\author{
Edilson Nunes Pollnow ${ }^{1}$, Alice Gonçalves Osório ${ }^{1}$, \\ Douglas Bezerra de Araújo ${ }^{2}$, Thais Andrezza dos Passos ${ }^{3}$, Daniel Souza ${ }^{3}$.
}

\footnotetext{
${ }^{1}$ Centro de Desenvolvimento Tecnológico - CDTec, UFPel - Universidade Federal de Pelotas, CEP: 96010-610, Pelotas, RS. e-mail: edilson.pollnow@hotmail.com,osorio.alice@gmail.com

${ }^{2}$ Faculdade de Engenharia Mecânica - FEMEC, UFU - Universidade Federal de Uberlândia, CEP: 38400-902, Uberlândia, MG. e-mail: dbaraujo@ufu.br

${ }^{3}$ Escola de Engenharia - EE, FURG - Universidade Federal do Rio Grande, CEP: 96203-900, Rio Grande, RS e-mail: thais.andrezza.passos@gmail.com,danielsouza@furg.br
}

\begin{abstract}
RESUMO
Os estudos na área da engenharia de superfícies recebem grande importância devido ao aprimoramento que as modificações superficiais fornecem aos materiais. Com um mercado global que tem a necessidade de proporcionar às peças e equipamentos maior vida útil com baixo custo, para suportar solicitações severas e assim manter seu alto desempenho, os tratamentos superficiais tornam o que se pensava impossível na realidade. Dentre os tratamentos superficiais que têm recebido atenção, e é objeto deste trabalho, temos o processo termoquímico de boretação. O processo de boretação consiste em saturar com boro a superfície de aços e ligas metálicas. Essa saturação fornece à superfície um incremento de propriedades não intrínsecas ao metal de base, tais como: dureza, resistência à abrasão e corrosão. Embora as propriedades dos aços boretados já tenham sido estudadas, os efeitos que o processo de boretação exerce nos aços durante ou posteriormente a processos de soldagem não são conhecidos. É objetivo deste trabalho avaliar a microestrutura e resposta mecânica de um aço de baixo carbono submetido ao tratamento de boretação pré e pós-soldagem GTAW. Os resultados apontaram uma má soldabilidade metalúrgica do aço de baixo teor de carbono com camada boretada, com a presença de trincas de solidificação. Já o aço soldado previamente à boretação apresentou um núcleo mais dúctil devido ao tratamento térmico submetido pós soldagem, porém com superfície de elevada dureza. Embora os valores de dureza do núcleo tenham caído drasticamente na zona soldada após este processo, a superfície destas amostras apresentou elevada dureza devido à camada boretada, o que pode ser considerado, principalmente em aplicações visando a resistência ao desgaste.
\end{abstract}

Palavras-chave: Boretação, GTAW, Microestrutura.

\begin{abstract}
Studies on surface engineering area are given great importance due to the improvement that surface modifications provide to materials. With a global market that has the need to provide parts and equipment with extended service life and low cost, to support stringent requests and thus maintain its high performance, surface treatments may bring what was impossible into reality. Among the surface treatments that have received attention recently, we have the thermochemical process of boriding. The boriding process consists of saturate the surface of steels and metal alloys with boron. This saturation provides an increase in the surface properties not inherent to the base metal, such as hardness, resistance to abrasion and corrosion. Although the properties of boriding steels have already been studied, the effects that the boriding process perform on the steel during or after the welding processes are not known. Hence, it is the purpose of this study to evaluate the microstructure of a low carbon steel treated with boriding before and after GTAW
\end{abstract}


welding. The results indicated poor metallurgical weldability of low carbon steel with boride layer, with the presence of solidification cracks. Nonetheless, the steel welded previous to boring treatment presented a more ductile nucleus, with harder surface. Although the values of hardness within the nucleus of the steel had dropped drastically at the welded zone after the boring, the surface of this steel showed higher values of hardness due to the boriding layer. This fact should be considered when applications where wear resistance is needed.

Keywords: Boriding, GTAW, Microstructure.

\section{INTRODUÇÃO}

Países com elevado índice de desenvolvimento utilizam amplamente a Engenharia de Superfícies em sua produção há muito tempo. A utilização de tratamentos superficiais nos materiais de engenharia pode melhorar suas propriedades tribológicas, bem como aumentar também a resistência à oxidação e corrosão, entre outros fatores.

Os tratamentos termoquímicos são tratamentos superficiais que consistem basicamente em aquecer a peça ou substrato em uma atmosfera rica nos elementos a que se deseja que ocorra a difusão, tais como: carbono, nitrogênio, boro, etc. Um destes tratamentos termoquímicos de superfícies que tem apresentado destaque é o processo de boretação. Boretação ou borotização é um importante tratamento termoquímico aplicado para melhorar a dureza superficial e resistência ao desgaste de ligas ferrosas e não ferrosas (1).

A Boretação envolve a difusão de átomos de boro na superfície de diferentes materiais ferrosos e não ferrosos a temperaturas que variam de $1123 \mathrm{~K}$ a $1273 \mathrm{~K}$ e com o tempo de exposição variando de 1 a $10 \mathrm{~h}$ ou mais (2).

Em materiais ferrosos, este tratamento termoquímico de difusão geralmente possui características de endurecimento superiores aos encontrados em processos convencionais como cementação, nitretação ou cromagem, devido à formação de uma única fase $\left(\mathrm{Fe}_{2} \mathrm{~B}\right)$ ou duplex $\left(\mathrm{FeB}+\mathrm{Fe}_{2} \mathrm{~B}\right)$, fases duras $(2,3,4)$ vide Figura 1.

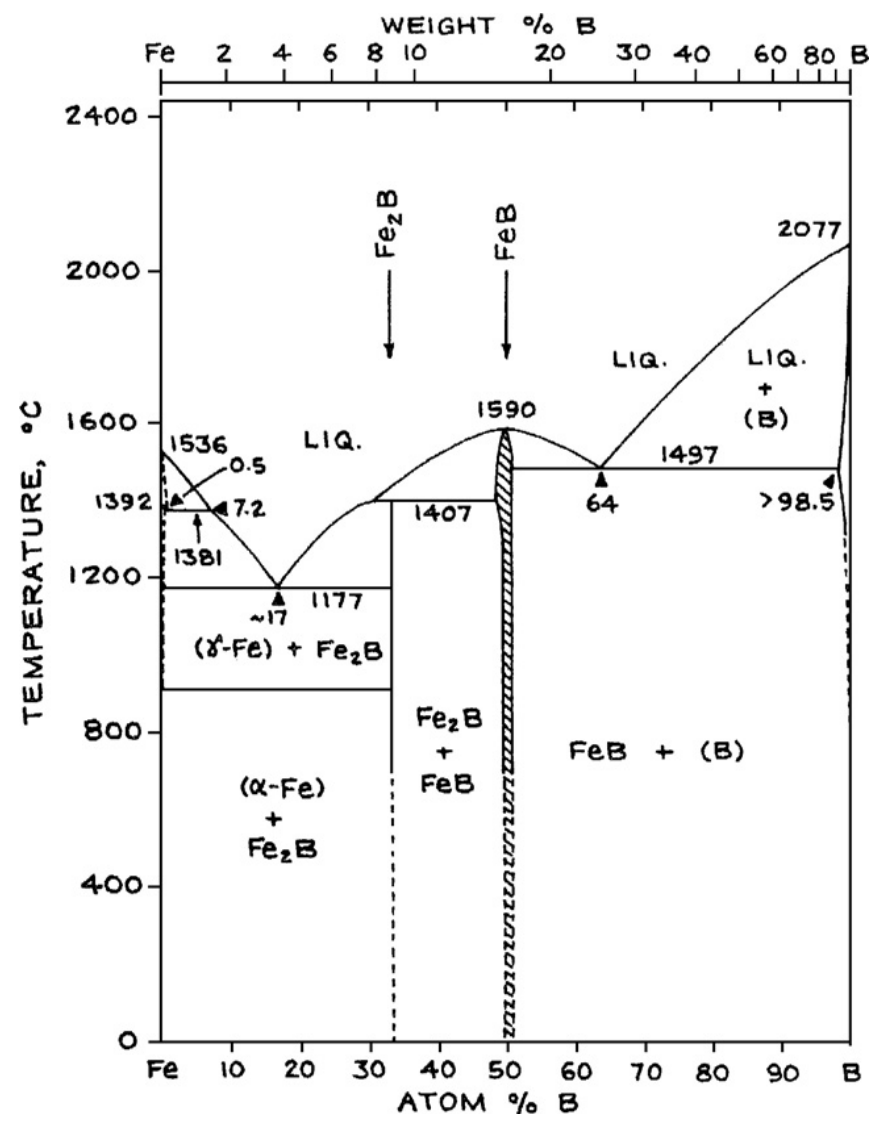

Figura 1: Diagrama de Fases Fe-B. Adaptado de Allaoui et. al. (4). 
A interface entre a região boretada e a região de difusão apresenta a forma de "dente de serra" em ferro puro, aços carbono e baixa liga e que vai se tornando plana à medida que o carbono e os elementos de liga são adicionados no aço. Os elementos de liga retardam o crescimento da região boretada por redução da difusividade do boro (5).

Boretação pode ser feita por meio de misturas de pós, sais, óxidos fundidos, bem como por meios de gasosos e por pastas (6). A Boretação em pó tem as vantagens de simplicidade e custo-benefício, em comparação com outros processos de boretação. Nesta técnica, o agente boretante na forma de pó é colocado dentro de uma caixa resistente ao calor e amostras são envolvidas neste pó sob esta atmosfera de gás inerte (7).

O grande problema de muitos tratamentos de superfície é a possibilidade de gerar dificuldades de soldagem posteriores devido à presença de elementos químicos que abaixam a linha solidus, e ocasionam defeitos de soldagem.

A soldagem é um dos processos de união mais importantes utilizados no setor automotivo, construção naval, construção civil, energético, aeroespacial e outras indústrias. Ela tem muitas vantagens competitivas em relação a outros métodos de união, tais como maior resistência do conjunto, baixo custo inicial, tempo de preparação reduzido e uma vasta gama de aplicações (8).

Entre os processos de soldagem existentes, o GTAW (Gas Tungsten Arc Welding) é tipicamente usado devido a precisão, estabilidade, excelente aparência da solda e qualidade em materiais avançados, finos ou ultrafinos $(9,10)$.

Neste contexto, se faz extremamente necessária a investigação da aplicação de processos que aperfeiçoem as propriedades de resistência ao desgaste e que possuam também a possibilidade da união por processos de soldagem com boa qualidade.

Atento a esta necessidade, é objetivo deste trabalho avaliar os efeitos do tratamento de boretação como processo de pré e pós-soldagem GTAW autógeno de um aço de baixo teor de carbono.

\section{MATERIAIS E MÉTODOS}

\subsection{TERMOQUÍMICO DE BORETAÇÃO}

Para a aplicação do tratamento de boretação, inicialmente as amostras de um aço de baixo teor de carbono do tipo SAE 1015 (ver composição química na Tabela 1) foram cortadas em duas diferentes condições: $70 \mathrm{~mm} \mathrm{x}$ $25 \mathrm{~mm}$ x $6 \mathrm{~mm}(\mathrm{C}$ x L x E) para o tratamento de boretação pré-soldagem, e $140 \mathrm{~mm}$ x 25 x $6 \mathrm{~mm}(\mathrm{C}$ x L x E) para o tratamento de boretação pós-soldagem.

Tabela 1: Composição química do aço selecionado.

\begin{tabular}{l|l|l|l|l}
\hline \multicolumn{5}{l}{ ELEMENTOS (\%) } \\
\hline $\mathrm{C}$ & $\mathrm{Si}$ & $\mathrm{Mn}$ & $\mathrm{P}$ & $\mathrm{S}$ \\
\hline 0,159 & 0,140 & 0,583 & 0,014 & 0,018 \\
\hline
\end{tabular}

Após o corte todas as amostras foram lixadas em via úmida utilizando-se lixas de SiC com diferentes granulometrias $(80$ - 1000 mesh) para remoção de contaminantes da superfície e para aumentar a área superficial da amostra em contato com o agente boretante.

As amostras foram colocadas em um cadinho e envoltas com $10 \mathrm{~mm}$ de agente boretante Ekabor ${ }^{\circledR} 1$ V2 e, posteriormente colocadas em um forno tubular INTI FT 1200 em atmosfera inerte. Utilizou-se uma taxa de aquecimento de $293 \mathrm{~K} / \mathrm{min}$ e resfriamento livre em atmosfera inerte dentro do forno. O agente boretante Ekabor ${ }^{\circledR} 1-\mathrm{V} 2$ apresenta composição aproximada de 5\%p B ${ }_{4} \mathrm{C}, 5 \% \mathrm{p} \mathrm{KBF} 4$ e 90\%p SiC (11). horas.

Para a boretação pré-soldagem, a temperatura utilizada foi de $1273 \mathrm{~K}$ com patamar de tempo de 2

Para o tratamento de boretação posterior a soldagem, foram utilizadas as temperaturas de $1123 \mathrm{~K}$ e $1273 \mathrm{~K}$ em iguais patamares de tempo de 2 horas.

Foram confeccionadas também amostras somente com o cordão de solda sob chapa, sem a adição de nenhum tratamento anterior ou posterior, para comparação das propriedades mecânicas com as amostras com 
tratamento.

Durante todo o tratamento de boretação foi utilizada atmosfera inerte de nitrogênio com vazões de 8 1/min para a purga do sistema de gases, e 6 1/min durante o restante do processo. Após o período de difusão, ocorreu o resfriamento livre das amostras em atmosfera inerte (no forno), a fim de evitar possíveis oxidações.

\subsection{PROCESSO GTAW AUTÓGENO}

Para a realização dos cordões de solda foi utilizado o processo de soldagem GTAW autógeno. A escolha por um processo autógeno deve-se ao fato deste não incluir metais de adição que poderiam afetar negativamente a microestrutura final do cordão, e estaria fora do escopo deste estudo. Foi utilizada como fonte de soldagem a fonte Fronius modelo TransPuls Synergic 4000.

Os parâmetros de soldagem são regulados no painel da fonte. A manipulação da tocha GTAW foi feita através de um robô MOTOMAN modelo HP20D. Este robô caracteriza-se por possuir seis graus de liberdade e suportar uma capacidade máxima de $20 \mathrm{Kg}$ em sua extremidade.

A programação do robô foi realizada de forma bem simplificada, consistindo em: movimentação até o local de início da solda, abertura do pré-gás, abertura do arco elétrico, movimentação com a velocidade de soldagem definida, fechamento do arco elétrico, pós-gás e afastamento do local da solda. Todos os comandos de soldagem são enviados à fonte de soldagem. Fez-se necessários determinar os parâmetros que mais se adequassem ao aço de baixo carbono submetido à boretação. Os parâmetros impostos ao processo encontram-se na Tabela 2.

Tabela 2: Parâmetros de soldagem.

\begin{tabular}{c|c|c|c|c|c|c}
\hline $\begin{array}{c}\text { I (CC-) } \\
(\mathrm{A})\end{array}$ & $\begin{array}{c}\text { V. SOLD. } \\
(\mathrm{CM} . \mathrm{MIN}-1)\end{array}$ & $\begin{array}{c}\text { GÁS DE } \\
\text { PROTEÇÃO }\end{array}$ & $\begin{array}{c}\text { VAZÃO } \\
(\text { L. MIN-1 })\end{array}$ & $\begin{array}{c}\varnothing \text { ELETRODO } \\
(\mathrm{MM})\end{array}$ & $\begin{array}{c}\text { ÂNGULO DO } \\
\text { ELETRODO }\left({ }^{\circ}\right)\end{array}$ & $\begin{array}{c}\text { LA } \\
(\mathrm{MM})\end{array}$ \\
\hline 120 & 15 & Argônio & 12 & 1,6 & 60 & 3,2 \\
\hline
\end{tabular}

I = corrente; V.Sold. $=$ Velocidade de soldagem; $\varnothing$ Eletrodo = diâmetro do eletrodo; $L a=$ Comprimento de arco.

Duas condições foram estudadas: a primeira onde o processo de soldagem foi realizado após o tratamento de boretação e a segunda quando a soldagem foi realizada previamente a etapa de boretação. Para este estudo não foram realizados nenhum tipo de preparação de junta; em ambos os casos foi realizado um passe de solda único e sobre chapa, respeitando os mesmos parâmetros de soldagem.

\subsection{CARACTERIZAÇÃO DAS AMOSTRAS}

\subsubsection{Microscopia Óptica}

Para avaliação do comportamento microestrutural das amostras utilizou-se a técnica de microscopia óptica. As análises foram realizadas em um microscópio ótico de luz refletida com sistema de análise de imagens Olympus GX 51S com aquisição de imagens digital.

Para a análise microscópica, foi necessária a realização de uma preparação metalográfica das amostras conforme ASTM E3-11 (12). Esta contou com as etapas de corte, embutimento, lixamento, polimento e ataque químico das amostras, com uma solução de Nital 3\%. O lixamento e o polimento foram realizados na Politriz semi-automática MiniMet ${ }^{\circledR} 250$ com lixas de granulometria 80, 180, 320, 400, 600, 1200 e 2000 mesh e para a etapa de polimento com suspensão de alumina com granulometria de $0,1 \mu \mathrm{m}$ com carga de 27 $\mathrm{N}$ por corpo de prova.

Para a avaliação da espessura de camada de boretos foi utilizada a metodologia sugerida por Campos et al. (13), onde é feita a média sobre o somatório de 10 medidas dos boretos, desde o de menor ao de maior espessura, conforme Figura 2. 


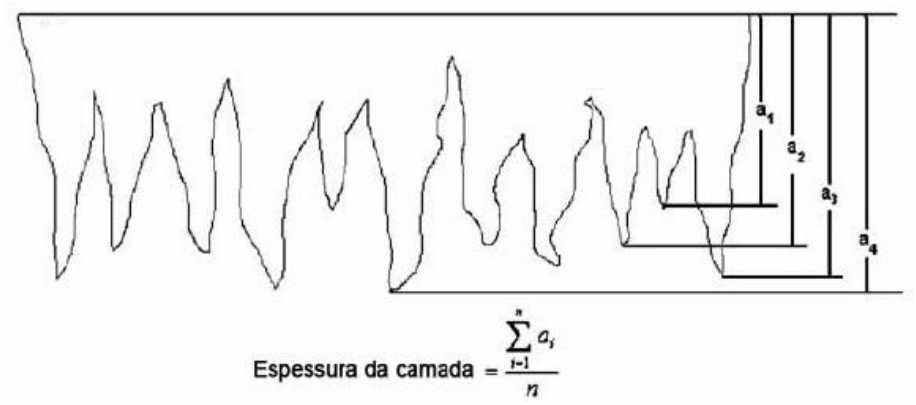

Figura 2: Esquema da medição da camada de boretos.

\subsubsection{Microdureza}

Para avaliar a dureza das amostras boretadas e soldadas foi realizado o ensaio de microdureza Vickers em um microdurômetro marca Shimadzu, modelo MVT-2T, segundo a norma ASTM E384-11E1 (14), empregando uma carga de $100 \mathrm{~g}$ para as zonas do aço SAE 1015 e uma carga de $50 \mathrm{~g}$ para a zona de boretos, pois estes por possuírem uma dureza muito elevada trincam ao serem submetidos a cargas mais elevadas, com um tempo de indentação de 14 segundos e distância entre indentações de 2,5 a 3 diagonais de indentação. Foram realizadas 20 medições por corpo de prova, 10 no aço SAE 1015 e 10 na região de boretos.

\subsubsection{Difração de Raios-X}

Utilizou-se da técnica de difratometria de raios-X para verificação das fases presentes nas amostras boretadas após soldagem.

Para esta análise foi utilizado um difratômetro de raios-X marca Philips, modelo X'Pert MPD. Equipado com monocromador de grafite e ânodo fixo. As análises foram coletadas via radiação de $\mathrm{Cu}-\mathrm{K} \alpha$ $\left(\lambda=1,5406 \AA\right.$ ) com ângulo de varredura $(2 \theta)$ na faixa entre $30-80^{\circ}$. Como referência foi utilizado o banco de dados JCPDSICDD (Joint Committee on Powder Diffraction Standards - International Centre for Diffraction Data), fichas 76-0092 e 75-101062.

\section{RESULTADOS E DISCUSSÕES}

\subsection{BORETAÇÃO PRÉ-SOLDAGEM}

Após a realização do processo de boretação, obteve-se uma camada boretada bem uniforme ao longo da superfície metálica, caracterizada por sua morfologia "dente de serra" com espessura média de camada de $78,35 \mu \mathrm{m}$ e dureza de 1453,5 HV0,05, conforme figura 3. Esses resultados corroboram com os estudos de Carrera-Espinoza et. al., Gómez-Vargas et. al., Kartal et. al [2,3,15].

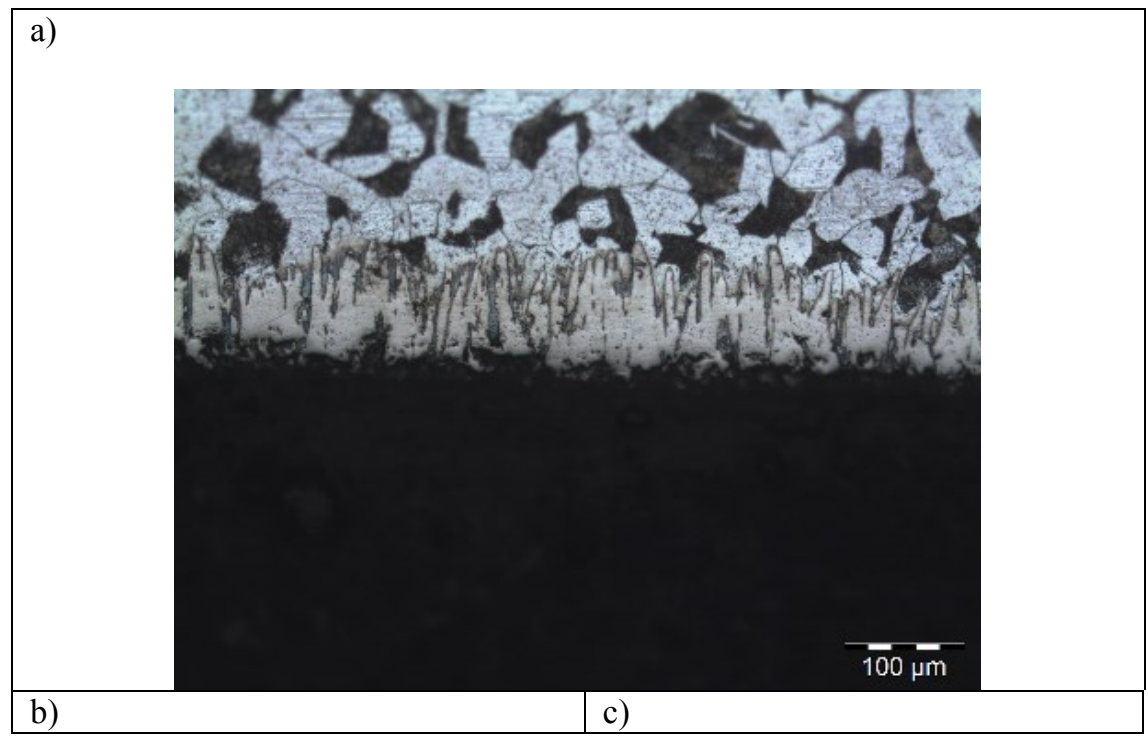




\begin{tabular}{|c|c|c|c|}
\hline \multirow[b]{2}{*}{ 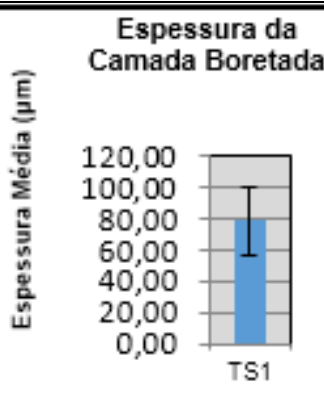 } & \multicolumn{3}{|c|}{$\begin{array}{c}\text { Microdureza da } \\
\text { Camada Boretada }\end{array}$} \\
\hline & 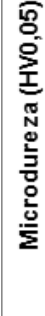 & $\begin{array}{r}1800,00 \\
1500,00 \\
1200,00 \\
900,00 \\
600,00 \\
300,00 \\
0,00\end{array}$ & $I$ \\
\hline
\end{tabular}

Figura 3: a) Camada Boretada a $1273 \mathrm{~K}$; b) Espessura da Camada Boretada a $1273 \mathrm{~K}$; e c) Microdureza da Camada Boretada a $1273 \mathrm{~K}$.

As micrografias da amostra em que ocorreu a solda sobre a camada boretada (Figura 4) demonstram a ocorrência de defeitos ao longo da zona fundida. Observa-se trincas de solidificação ao longo da zona de fusão, causadas por filmes líquidos de possíveis microssegregações de boro durante a etapa de solidificação. A associação de trincas de solidificação com a presença de boro ou outros elementos é mencionada por Modenesi et.al. [16], porém não é mencionado claramente a forma como os boretos influenciam na presença destas trincas.

A presença de grãos finos na zona fundida $(\mathrm{ZF})$ indica uma rápida extração de calor que pode ser responsável também por facilitar a ocorrência das trincas. As trincas se desenvolvem ao longo dos contornos de grão ferritícos e se propaga até o final da zona fundida. A microestrutura da ZF é formada por grãos de Ferrita Poligonal, Ferrita Intragranular Poligonal e regiões de Ferrita Widmanstätten de Contorno de Grão.

A Zona Termicamente Afetada (ZTA) da amostra (Figura 4b) apresentou Ferrita Poligonal de Contorno de Grão, Ferrita Intragranular Poligonal e Perlita em sua microconstituição. Uma morfologia muito próxima a existente no metal de base.

O metal de base da amostra (Figura 4c) apresenta em sua morfologia Ferritas Poligonais de Contorno de Grão (área clara) e regiões Perliticas (área escura).
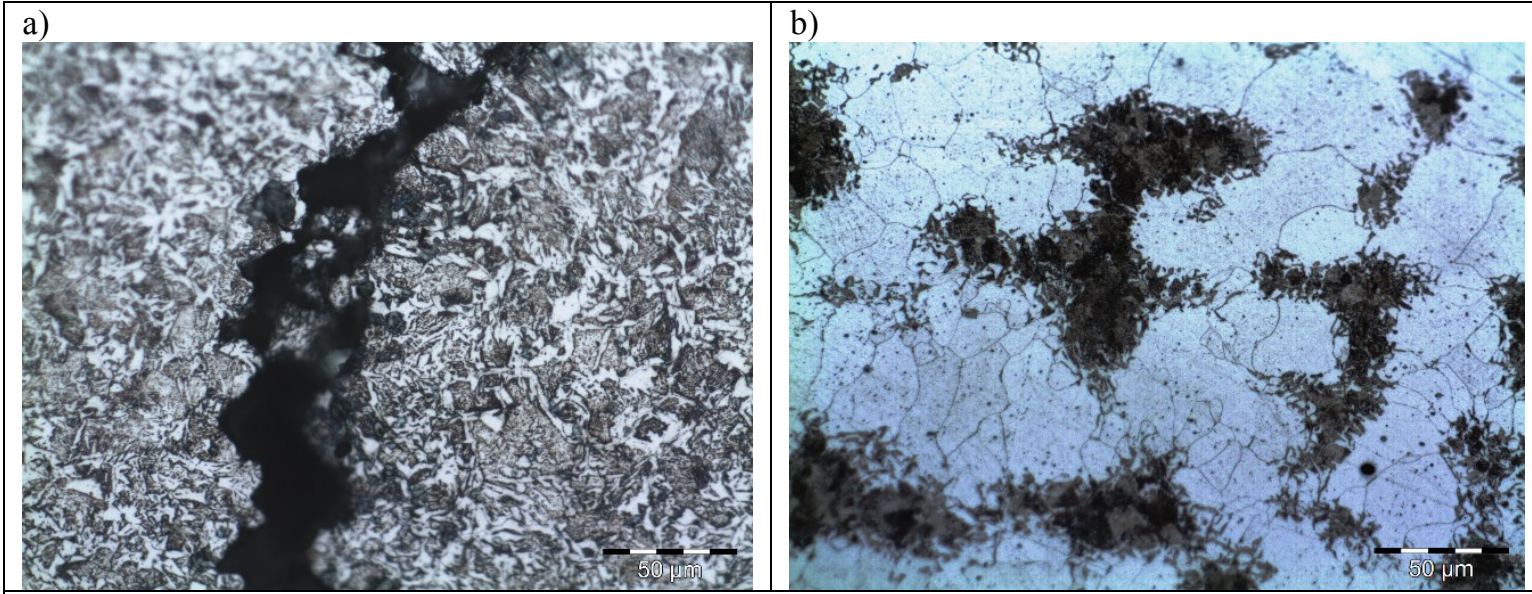

c) 


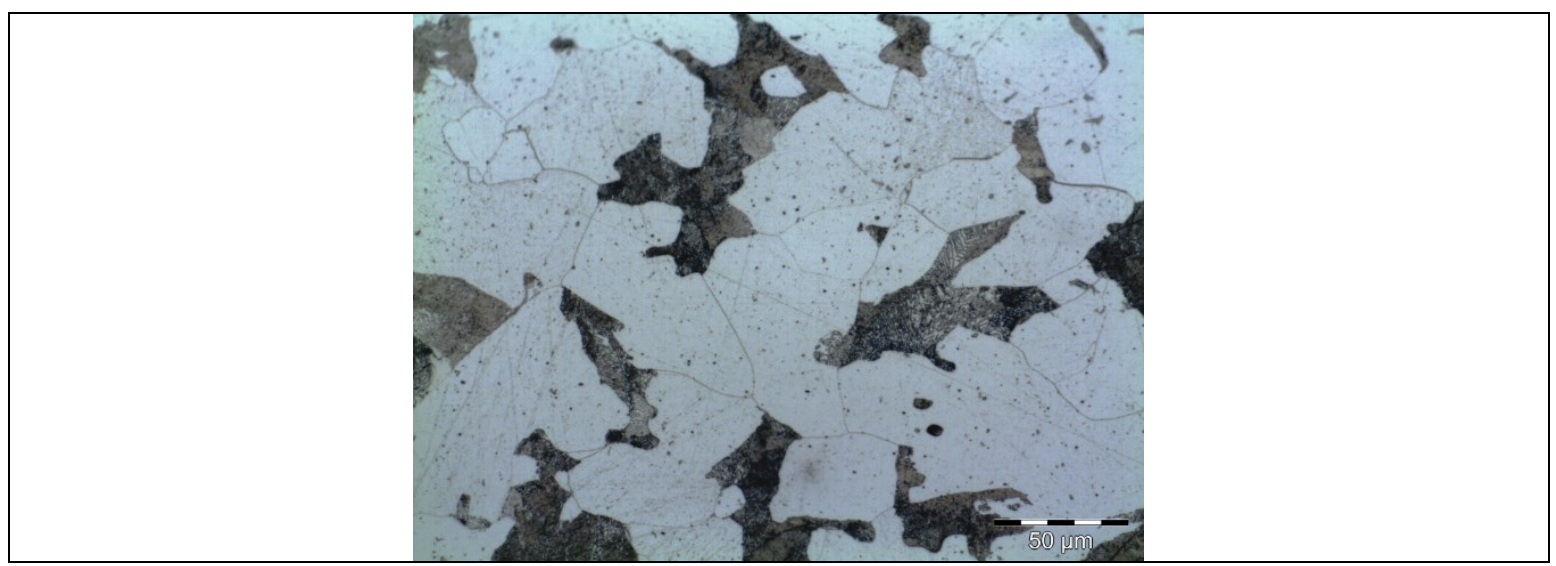

Figura 4: Amostra com Boretação pré-soldagem. a) ZF; b) ZTA e c) MB.

A soldagem sobre a camada boretada tem além das alterações microestruturais, uma influência muito grande na microdureza, principalmente na região da zona fundida como pode ser visto na Figura 5. Esse fato acontece devido a diluição da região boretada para a zona de fusão e pode influenciar positivamente para a formação das trincas de solidificação observadas.

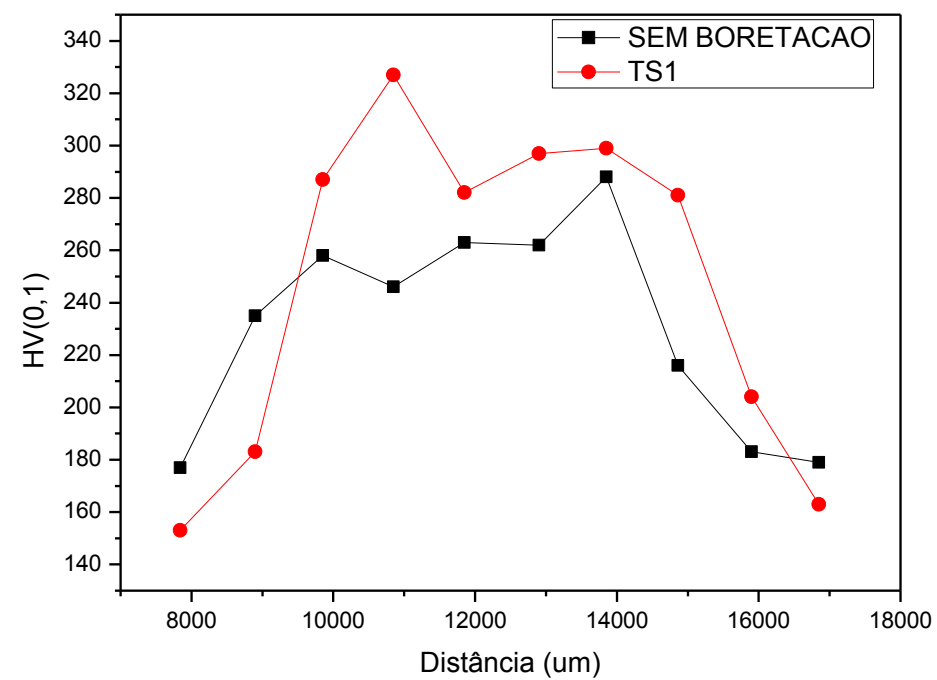

Figura 5: Microdureza das amostras sem tratamento e soldada após boretação.

\subsection{BORETAÇÃO PÓS-SOLDAGEM}

O processo de boretação realizado após a etapa de soldagem GTAW produziu camadas boretadas com a morfologia "dente de serra" como descrita anteriormente, com espessuras de camada médias de 19,70 $\mu \mathrm{m}$ e $44,01 \mu \mathrm{m}$ para as amostras boretadas a $1123 \mathrm{~K}$ e $1273 \mathrm{~K}$ respectivamente, e microdurezas respectivas de 1435,10 HV0,05 e 1423,30 HV0,05 (vide Figura 6). 


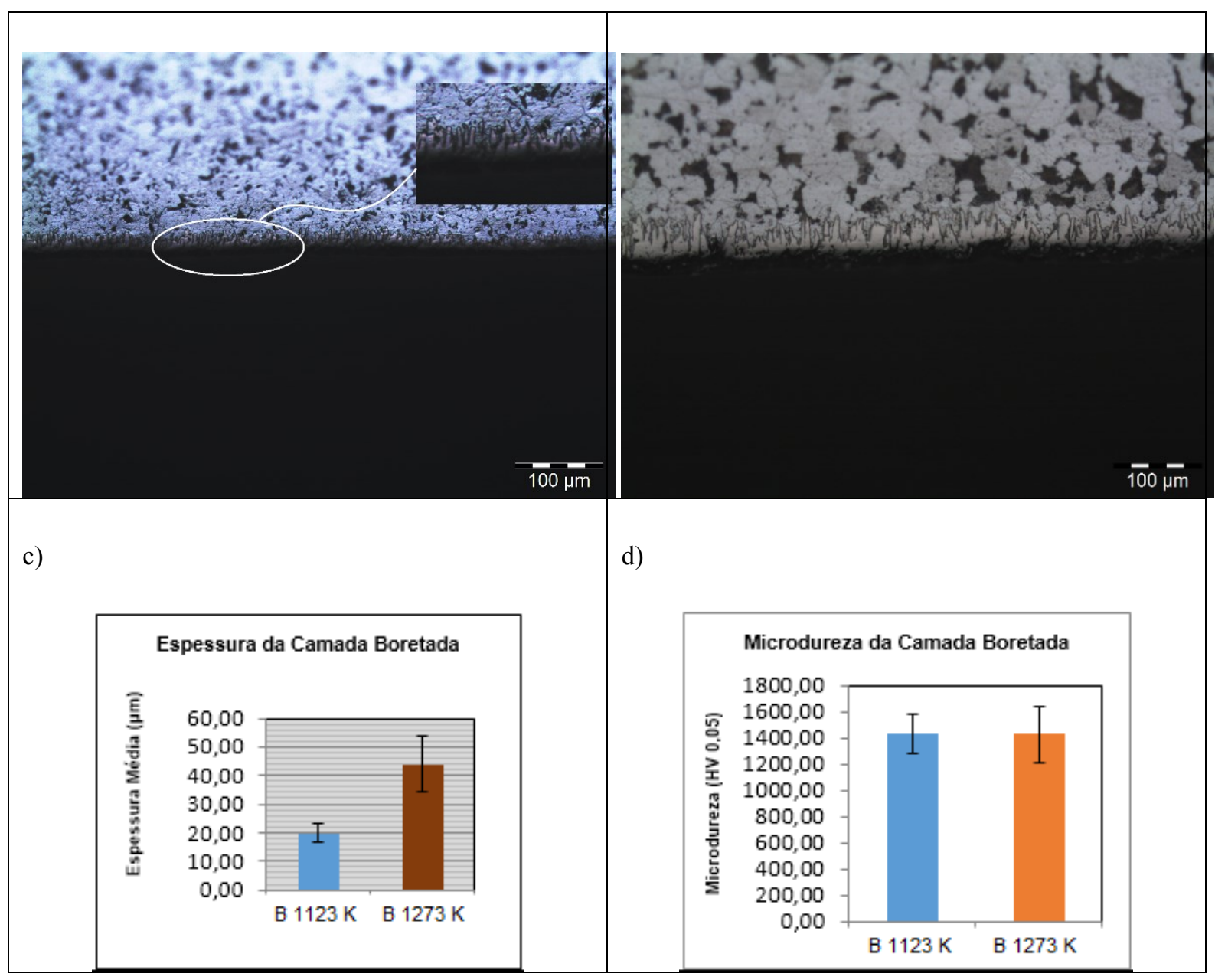

Figura 6: a) Camada boretada a $1123 \mathrm{~K}$; b) Camada boretada a $1273 \mathrm{~K}$; c) Espessura de camada para ambas amostras; e d) Microdureza da camada boretada de ambas amostras.

As micrografias apresentadas na Figura 7 apontam variações da microestrutura das amostras submetidas à soldagem com posterior boretação a $1123 \mathrm{~K}$ e as amostras submetidas soldagem e boretação a 1273 K. Quando enfocamos na ZF é comum a presença de Ferritas Poligonais de Contorno de Grão e Perlita para ambas as amostras. Porém a amostra boretada a $1123 \mathrm{~K}$ apresenta ainda Ferritas de Widmanstätten de Contorno de Grão.

Na ZTA de ambas as amostras os microconstituintes apresentados são os mesmos, Ferritas Poligonais e Perlitas. A mesma microestrutura que constitui o metal de base.

Observa-se, ainda, um alto crescimento de grão nas amostras boretadas a $1273 \mathrm{~K}$ em relação àquelas boretadas a $1123 \mathrm{~K}$, que pode significar um decaimento nas propriedades mecânicas das primeiras.

Os resultados de microdureza (Figura 8) comprovam a perda de resistência mecânica. É possível observar o aumento da ductilidade das amostras boretadas após o processo de soldagem em comparação às amostras sem tratamento. Quando tomamos por exemplo a zona de fusão temos uma microdureza média de 264 HV0,1 para a amostra soldada e sem tratamento, 144 HV0,1 e 123,5 HV0,1 para as amostras soldadas e boretadas a $1123 \mathrm{~K}$ e $1273 \mathrm{~K}$ respectivamente, situação semelhante a observada por Meric et. al.[17] . É importante ressaltar que os valores de microdureza foram obtidos longe da zona de boretos, fator importante, pois se obtido em uma região próxima ou de interface, os valores poderiam não caracterizar a resistência mecânica do metal de base.

Observa-se também uma certa linearidade entre os valores de microdureza para as regiões do metal de base, ZTA e ZF da amostra soldada e boretada a $1273 \mathrm{~K}$. Esses resultados corroboram com as análises de microscopia que mostram que as amostras estão passando por processos de recozimento a $1123 \mathrm{~K}$ e normalização a $1273 \mathrm{~K}$. 


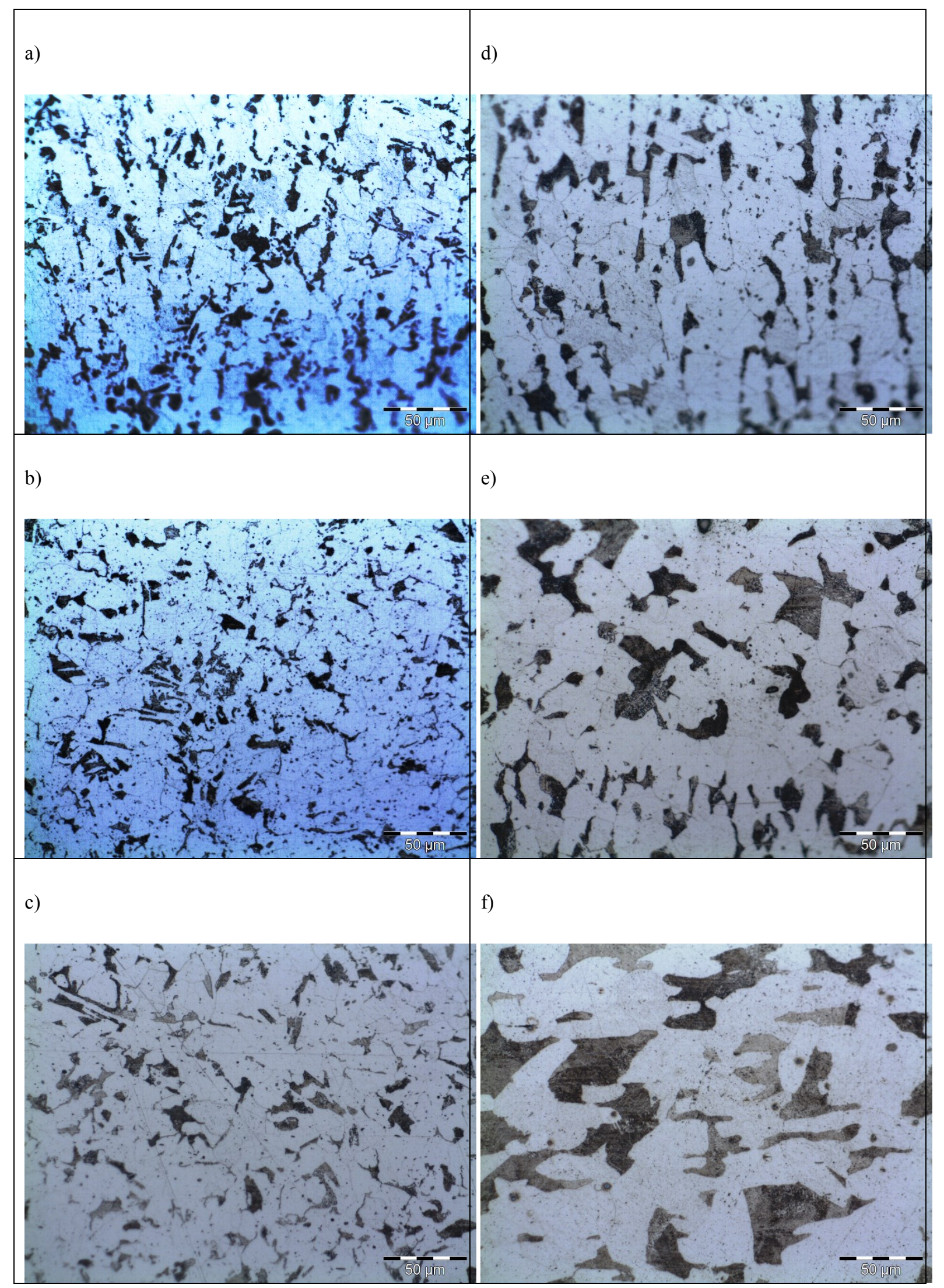

Figura 7: Micrografias das amostras: a) ZF amostra boretada a $1123 \mathrm{~K}$; b) ZTA boretada a $1123 \mathrm{~K}$; c) MB boretada a $1123 \mathrm{~K}$; d) ZF boretada a $1273 \mathrm{~K}$; e) ZTA boretada a $1273 \mathrm{~K}$ e f) MB boretada a $1273 \mathrm{~K}$. 


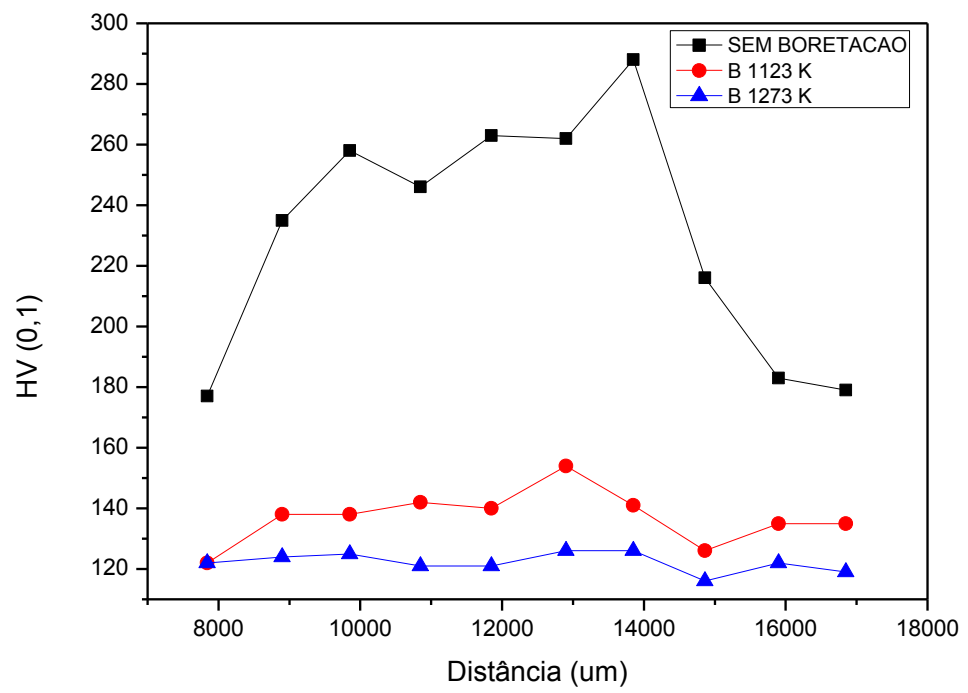

Figura 8: Microdureza das amostras soldadas sem boretação, boretadas a $1123 \mathrm{~K}$ e boretadas a $1273 \mathrm{~K}$.

Os difratogramas de raios-X (Figura 9) para ambas as temperaturas de boretação, apontam a formação de duas fases de boretos na superfície do cordão de solda, as fases $\mathrm{FeB}$ e $\mathrm{Fe}_{2} \mathrm{~B}$. Nos difratogramas observa-se uma menor intensidade da fase $\mathrm{FeB}$, inclusive com alguns picos de menor intensidade se confundido com o ruído, isto pode ser interpretado como um indicativo da presença desta fase em menor proporção em relação a fase $\mathrm{Fe}_{2} \mathrm{~B}$.

A presença desta dupla fase corrobora com a hipótese de que as trincas de solidificação se propiciaram a partir da segregação dos boretos, pois conforme o diagrama $\mathrm{Fe}-\mathrm{B}$ a temperatura de fusão quando coexistem as fases $\mathrm{FeB}$ e $\mathrm{Fe}_{2} \mathrm{~B}$ é de $1680 \mathrm{~K}$, esta é inferior a temperatura de fusão do aço SAE 1015 que é de aproximadamente $1780 \mathrm{~K}$.

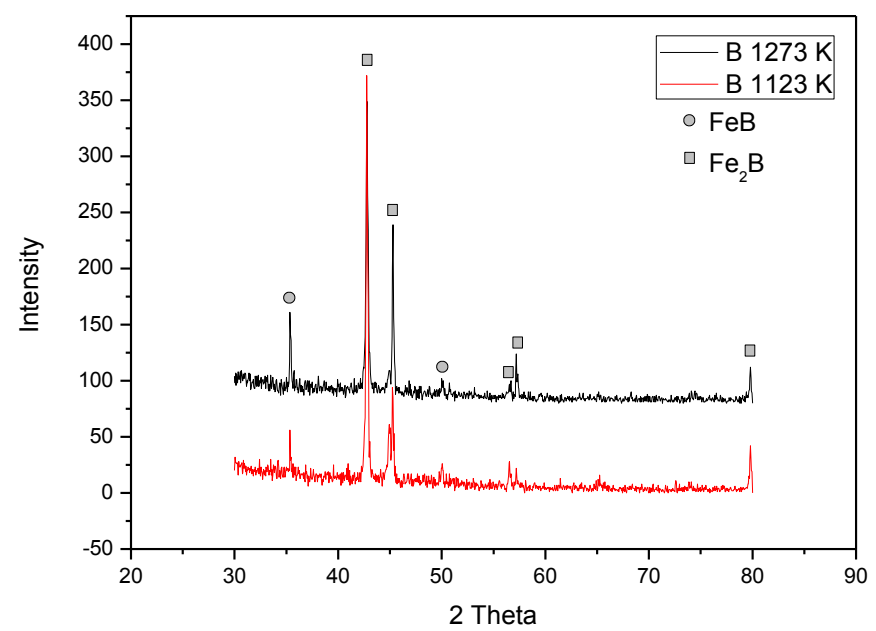

Figura 9: Difratogramas de Raios- $X$ das camadas boretadas pós-soldagem.

\section{CONCLUSÕES}

Após uma análise cuidadosa da amostra submetida ao processo de boretação e posteriormente ao processo de soldagem GTAW, percebe-se que a camada boretada influencia negativamente na soldabilidade do aço de 
baixo carbono SAE 1015, ocasionando a presença de trincas de solidificação devido a uma aceleração da solidificação do cordão de solda e a presença de fases líquidas abaixo da linha solidus do aço.

Para a condição soldada e posteriormente boretada, a nucleação e crescimento dos grãos apontam a ocorrência dos processos de recozimento nas amostras boretadas a $1123 \mathrm{~K}$ e de normalização nas amostras boretadas a $1273 \mathrm{~K}$, uma vez que o aumento de temperatura dos tratamentos foi determinante para as alterações microestruturais.

Embora valores de dureza tenham decrescido drasticamente na ZTA e ZB após este processo, a superfície destas amostras apresentaram elevada dureza devido à camada boretada. Entretanto, embora a dureza das amostras tenha decrescido, os valores de microdureza da zona de boretos de ferro são imensamente superiores às regiões do aço SAE 1015. Portanto, este tipo de condição de soldagem aliado ao processo de boretação deve ser avaliada com parcimônia quando aplicado em serviço, uma vez que deverá apresentar resultados satisfatórios em aplicações visando a resistência ao desgaste.

\section{AGRADECIMENTOS}

Os autores agradecem a CAPES (Coordenação de Aperfeiçoamento de Pessoal de Nível Superior) pelo apoio financeiro.

\section{BIBLIOGRAFIA}

[1] CULHA, O., TOPARLI, M., SAHIN, S., et al. "Characterization and determination of FexB layers' mechanical properties", Journal of materials processing technology, n. 206, pp. 231-240, December, 2007.

[2] CARRERA-ESPINOZA, R., FIGUEROA-LÓPEZ, U., MARTÍNEZ-TRINIDAD, J., et al., "Tribological behavior of borided AISI 1018 steel under linear reciprocating sliding conditions", Wear, n.362-363, pp. 1-7, September, 2016.

[3] GÓMEZ-VARGAS, O. A., SOLIS-ROMERO, J., FIGUEROA-LÓPEZ, U., et al., "Boro-nitriding coating on pure iron by powder-pack boriding and nitriding processes", Materials Letters, n. 176, pp. 261264, April, 2016.

[4] ALLAOUI, O., BOUAOUADJA, N., SAINDERNAN, G. "Characterization of boronized layers on a XC 38 steel”, Surface \& Coatings Technology, n. 201, pp. 3475-3482, September, 2006.

[5] PETROVA, R. S., SUWATTANANONT, N., SAMARDZIC, V. "The effect of boronizing on metallic alloys for automotive applications", Journal of Materials Engineering and Performance, n.17, pp. 340-345, February,2008.

[6] CAMPOS-SILVA, I., BALANKIN, A. S., SIERRA, A. H., et al., "Characterization of rough interfaces obtained by boriding", Applied Surface Science, n. 255, pp. 2596-2602, August, 2008.

[7] ŞAHIN, S. "Effects of boronizing process on the surface roughness and dimensions of AISI 1020, AISI 1040 and AISI 2714", Journal of materials processing technology, n.209, pp. 1736-1741, April, 2008.

[8] GARCÍA-GARCÍA, V., CAMACHO-ARRIAGA, J. C., REYES-CALDERÓN, F. "A simplified elliptic paraboloid heat source model for autogenous GTAW process", International Journal of Heat and Mass Transfer, n. 100, pp. 536-549, April, 2016.

[9] JIN, Z., LI, H., JIA, G., et al., "Dynamic nonlinear modeling of 3D weld pool surface in GTAW", Robot Robotics and Computer-Integrated Manufacturing, n. 39, pp. 1-8, December, 2015.

[10] WU, C. S., CHEN, J., ZHANG, Y. M. "Numerical analysis of both front- and back-side deformation of fully-penetrated GTAW weld pool surfaces", Computational Materials Science, n. 39, pp. 635-642, August, 2006.

[11] GUNES, I., ULKER, S., TAKTAK, S. "Investigation of tribological behaviour of plasma paste boronized of AISI 8620, 52100 and 440c steels", Materials and Design, n. 32, pp. 2380-2386, November, 2010.

[12] AMERICAN SOCIETY FOR TESTING AND MATERIALS. ASTM E3-11 - "Standard Guide for Preparation of Metallographic Specimens 1", ASTM International, West Conshohocken, PA, 2011.

[13] CAMPOS, I., FARAH, M., LÓPEZ, N., et al., "Evaluation of the tool life and fracture toughness of cutting tools boronized by the paste boriding process", Applied Surface Science, n. 254, pp. 2967-2974, October, 2007.

[14] AMERICAN SOCIETY FOR TESTING AND MATERIALS. ASTM E384 - "Standard Test Method for Knoop and Vickers Hardness of Materials1". ASTM International, West Conshohocken, PA, 2012. 
[15] KARTAL, G., TIMUR, S., ERYILMAZ, O. L., et al., "Influence of process duration on structure and chemistry of borided low carbon steel”, Surface \& Coatings Technology, n. 205, pp. 1578-1583, August, 2010.

[16] MODENESI, P. V., MARQUES, P. J., SANTOS, D. B. "Fissuração em Juntas Soldadas" In: Introdução a metalurgia da soldagem, 1 ed., cap. 7, Belo Horizonte, UFMG - Universidade Federal de Minas Gerais, 2012

[17] MERIC, C., SAHIN, S., UZKUT, M. "Investigation of effect of boronizing on welding zone", Science and Technology of Welding and Joining, v. 7, n. 2, pp. 107-110, September, 2001. 